\title{
Author Correction: On the causes of geographically heterogeneous parallel evolution in sticklebacks
}

\author{
Bohao Fang (D), Petri Kemppainen (D), Paolo Momigliano (D), Xueyun Feng (D) and Juha Merilä (D) \\ Correction to: Nature Ecology \& Evolution https://doi.org/10.1038/s41559-020-1222-6, published online 22 June 2020.
}

In the version of this Article originally published, chromosomal labels were inadvertently switched for LD-clusters 15-20 in Extended Data Figs. 1 and 7, and in one sentence in the main text for LD-cluster 19. The errors were introduced when erroneously sorting chromosomes to produce the figures by roman (rather than numerical) numbers in the $\mathrm{R}$ code.

In Extended Data Fig. 1, the chromosomal names shown on the $x$ axes of the upper and middle plots for LD-clusters 15-20 were ' 8 ', ' 9 ', ' 5 ', ' 5 ', ' 5 ' and ' 5 ' but should have been '7', ' 8 ', '9', '9', '9' and '9', respectively.

In Extended Data Fig. 7, the chromosomal names in the 'Located Chromosome' column for LD-clusters 15-20 were 'Chr. VIII', 'Chr. IX', 'Chr. V', 'Chr. V', 'Chr. V' and 'Chr. V' but should have been 'Chr. VII', 'Chr. VIII,' 'Chr. IX', 'Chr. IX', 'Chr. IX' and 'Chr. IX’, respectively.

In the Results subsection 'Trans-oceanic marine-freshwater parallelism', the sentence 'In addition, we also found a putative novel inversion on chromosome V (LD-cluster 19, 241 loci) that was not associated with marine-freshwater differentiation (Extended Data Fig. 1 and Extended Data Fig. 7).' should have been 'In addition, we also found a putative novel inversion on chromosome IX (LD-cluster 19, 241 loci) that was not associated with marine-freshwater differentiation (Extended Data Fig. 1 and Extended Data Fig. 7).'

All errors had no effect on the results and conclusions, and have now been corrected.

Published online: 22 April 2021

https://doi.org/10.1038/s41559-021-01447-7

(C) The Author(s), under exclusive licence to Springer Nature Limited 2021 